\title{
BIENESTAR PSICOLÓGICO EN PAREJAS Y CODEPENDENCIA EN LA ETAPA DE LA JUVENTUD
}

\section{PSYCHOLOGICAL WELL-BEING IN COUPLES AND CODEPENDENCY IN THE YOUTH STAGE}

\author{
GRACE LATORRE VACA ${ }^{1}$ \\ MARIE-FRANCE MERLYN SACOTO \\ AGUSTÍN DOUSDEBÉS BOADA ${ }^{3}$
}

Recibido: 15 de mayo de 2017 Aceptado: 29 de agosto de 2017

\footnotetext{
${ }^{1}$ Pontificia Universidad Católica del Ecuador, Facultad de Psicología, Quito, Ecuador (galatorre@puce.edu.ec).

${ }_{2}^{2}$ Pontificia Universidad Católica del Ecuador, Facultad de Psicología, Quito, Ecuador (mfmerlyns@puce.edu.ec).

${ }_{3}^{3}$ Pontificia Universidad Católica del Ecuador, Facultad de Psicología, Quito, Ecuador (adousdebes@puce.edu.ec).
} 



\section{BIENESTAR PSICOLÓGICO EN PAREJAS Y CODEPENDENCIA EN LA ETAPA DE LA JUVENTUD}

\section{PSYCHOLOGICAL WELL-BEING IN COUPLES AND CODEPENDENCY IN THE YOUTH STAGE}

Grace Alexandra Latorre Vaca, Marie-France Merlyn Sacoto y Agustín Dousdebés Boada

PALABRAS CLAVES: bienestar psicológico, codependencia,juventud, pareja.

KEY WORDS: mental well-being, codependency, youth, couple.

\section{RESUMEN}

El propósito de la investigación fue determinar si el bienestar psicológico y el establecimiento de relaciones de pareja codependientes, durante la juventud, se encuentran relacionados. El estudio contó con una muestra estratificada conformada por 549 estudiantes de 17 a 27 años de edad, pertenecientes a la Pontificia Universidad Católica del Ecuador, PUCE. Se aplicó la Escala de Bienestar Psicológico (EPB), para medir el estado de bienestar psicológico subjetivo de los jóvenes y el Instrumento de Codependencia (ICOD) fue usado para determinar la existencia de relaciones de dependencia. Los resultados reflejan que existe una relación inversa entre ambas variables, dado que los participantes de este estudio con mayor bienestar psicológico presentan una menor incidencia de involucrarse en relaciones con patrón de codependencia. 


\section{ABSTRACT}

The purpose of the research was to determine if psychological well-being and the establishment of couple codependent relationships during the youth stage are related. The study involved a stratified sample of 549 students between 17 and 25 years old, belonging to the Catholic University of Ecuador - PUCE. Psychological Well-Being Scale (EPB) was applied to measure the subjective psy- chological well-being of young people and Codependency Instrument (ICOD) was used as evidence to establish the existence of codependent relationships. The results show that there is an inverse relationship between both variables because the participants of this study with greater psychological well-being have a lower incidence of involvement in relations with codependency patterns.

\section{BIENESTAR PSICOLÓGICO, RELACIÓN DE PAREJA Y CODEPENDENCIA}

\section{Bienestar mental}

No existe un modelo teórico referencial único en el tema del bienestar mental o psicológico. Por un lado, el modelo más empleado es el de Ryff (Ryff \& Singer, 1998a, 1998b) que propone que este concepto se articula alrededor de seis factores: auto aceptación, crecimiento personal, propósitos de vida, relaciones positivas con otros, dominio del medio ambiente y autonomía. Otros autores, como Fernández-Molina (2015), subrayan que desde diferentes teorías se ha conceptualizado el bienestar psicológico, definiéndolo como la satisfacción de los derechos y el buen trato, o como un concepto ligado a la salud, y también desde puntos de vista sociales (bienestar identificado con condiciones de vida y de trabajo) o felicidad (desde la psicología positiva).

Casullo (2002), por su parte, define al bienestar mental como un constructo de tres componentes: 1) Estados emocionales (afectos positivos y negativos), 2) Componente cognitivo (bienestar que deriva del procesamiento de las experiencias vitales) y 3) Relaciones vinculares. Estos componentes interactúan entre sí dando una sensación subjetiva de bienestar: la interpretación positiva de estados emocionales, el procesamiento cognitivo adecuado de las experiencias vitales y la existencia de relacio- 
nes vinculares satisfactorias derivarán en un sentido de bienestar mental, lo que a su vez permite un correcto ajuste de la persona en sus vivencias cotidianas (Pérez Padilla, 2010).

Por último, la Organización Mundial de la Salud (2013) define a la salud mental como

Un estado de bienestar en el cual el individuo es consciente de sus propias capacidades, puede afrontar las tensiones normales de la vida, puede trabajar de forma productiva y fructífera y es capaz de hacer una contribución a su comunidad.

Sin embargo, independientemente de la teoría, y así como lo concluye Fernández-Abascal (2009), lo que es clave en el concepto de bienestar psicológico son los componentes cognitivos y afectivos.

Por otro lado, si bien hay muchas definiciones del bienestar psicológico, es poca la información que se ha dado sobre los orígenes de este en el individuo. Se sabe que se desarrolla desde la interacción entre el sujeto y su entorno, y que existen entonces variables internas y externas que actúan en un bucle de retroalimentación, en donde el bienestar psicológico deriva de las vivencias exter- nas y su apreciación cognitiva y afectiva, y este a su vez determina poco a poco la calidad de las interacciones y de las experiencias posteriores. Fernández-Molina (2015) desarrolla un modelo ecológico del bienestar infantil (en términos de factores de riesgo y protección) en donde las condiciones propias del niño (características de la personalidad, resiliencia, capacidades cognitivas, condiciones de desarrollo normativo o no, necesidades básicas fisiobiológicas, afectivo emocionales, sociales y cognitivo-lingüísticas) interactúan con las sociales (sistema de protección social, sistema escolar, políticas y economía) y con las familiares (características de los padres y madres, de la situación familiar) para construir en el niño el llamado bienestar psicológico. Dentro de estas diversas condiciones, las emocionales necesarias para la estructuración psíquica han sido estudiadas desde hace varias décadas desde otras teorizaciones como la del apego (Ciccheti, Toth, \& Lynch, 1995; Bolwby, 1969, 1973) con conclusiones similares.

Varias visiones psicológicas han enfatizado que las condiciones emocionales, importantes para el establecimiento del bienestar mental, influyen también en el establecimiento de las relaciones sociales. Pero, ¿por qué estudiar esta relación en la juventud? 


\section{JUVENTUD Y RELACIÓN DE PAREJA}

Una de las tareas más importantes durante la etapa de la juventud es la de consolidar una pareja que se mantendrá durante la adultez como un referente de estabilidad emocional; en términos de Freud (citado en Erikson, 1971, pág. 111), la tarea de la adultez era: "amor y trabajo". Erik Erikson, por su parte, especificó aún más este punto y habló de las "crisis" de cada etapa -momentos cruciales del desarrollo asociados a edades específicas- en la cuales se adquiere un "sentido" que aporta a la identidad, o por el contrario el desarrollo se desvía dejando una impronta negativa en la personalidad. Si bien este autor describe crisis para cada etapa de la vida, ligó específicamente a la etapa de la juventud la crisis de "intimidad versus aislamiento". La intimidad, según Erikson (1968), tiene que ver con una forma profunda de encuentro con el otro: "el amor como devoción mutua supera los antagonismos inherentes a la polarización sexual y funcional y constituye la fuerza vital de la adultez joven" (pág.112).

Este encuentro con el otro es imposible si la propia persona no está consciente de su sí mismo, si no ha construido una identidad en la etapa previa, es decir en la adolescencia. Así, Erikson (1968) hace mucho énfasis en la construcción de un sentido de identidad en esa etapa, que está a su vez ligado al éxito en la adquisición de ciertas características de la personalidad a lo largo de la infancia; el asunto de la identidad es entonces transversal a lo largo de la vida. En la adolescencia, las relaciones de pareja no son de encuentro con el otro; son relaciones "de espejo", en donde se pretende más buscarse a sí mismo que relacionarse con alguien.

El establecimiento de la identidad al final de la adolescencia es un requisito para que se pueda alcanzar la intimidad en la relación de pareja (Erikson, 1971). De otra manera, el joven adulto puede llegar a generar un sentimiento de distanciamiento o aislamiento. Los Cimientos de una relación de pareja sana se construyen en etapas anteriores, pero la tarea de lograrla es para el adulto joven; en efecto, es en la juventud que se establecerán los encuentros verdaderos con el otro ser, y que se volverá importante que estos sean satisfactorios y contribuyan al bienestar mental de la persona, porque, como dice Craig (2009), "una parte de la identidad personal se adquiere por ser miembro de una pareja relativamente estable" (pág.451).

Las preguntas que surgen ante lo descrito anteriormente son: ¿qué pasa cuando esta importante tarea no se logra? Específicamente, ¿qué pasa cuando 
la relación de pareja no es sana? ¿Cómo repercute la calidad de la relación en el propio bienestar?

En un estudio realizado en Ecuador, a nivel de todo el país, (Instituto Nacional de Estadísticas y Censos, 2011) con mujeres de 15 años y más, se revela que 6 de cada 10 mujeres han vivido algún tipo de violencia; el $87,3 \%$ de estos casos fueron en sus relaciones de pareja; de ellas, $90 \%$ no se han separado. Si bien el tipo de violencia más común es la psicológica (53,9\%), 1 de cada 4 mujeres ha vivido violencia sexual. Además, el $30 \%$ de las mujeres ecuatorianas se casan justo en la etapa de la juventud, entre los 21 y los 25 años de edad.

Estas impactantes estadísticas hacen que nos cuestionemos las razones por las cuales mujeres jóvenes, de todos los estratos sociales, deciden mantener la vida en pareja pese a la atroz situación que están viviendo. El factor económico no puede ser una explicación puesto que la violencia de género según este estudio está generalizada (alrededor del 60\%) en los cinco quintiles de distribución del ingreso per cápita del hogar. El factor de educación puede ser una variable de peso, puesto que si bien en todos los niveles de instrucción la violencia de género sobrepasa el $50 \%$, en las mujeres que tienen menor nivel de educación la violencia llega al $70 \%$. Sin embargo, hablar de 1 de cada 2 mujeres en cualquier nivel de educación nos alerta sobre otro tipo de factores detrás del mantenimiento de relaciones patológicas de pareja. Es ahí que interviene la hipótesis de una construcción nociva de la relación de pareja.

\section{RELACIONES DE PAREJA Y CODEPENDENCIA}

Las relaciones cercanas se ubican en un continuo que va desde una relativa superficialidad, pasando por apertura y confianza relativas, hasta llegar a apertura y confianza absolutas. Esta última categoría suele asociarse al amor, a la intimidad y al matrimonio. Los autores Hogg \& Vaughan (2010) señalan que las relaciones cercanas están necesariamente marcadas por el amor que es una combinación de emociones, cognicio- nes y conductas propias de las relaciones íntimas.

Las relaciones cercanas están marcadas también por la interdependencia que consiste en una asociación interpersonal en la cual las personas influyen cada una en la vida de la otra y se involucran de manera conjunta en la realización de muchas actividades (Baron \& Byrne, 2005). La interdependecia es percibida positivamente por los autores, 
puesto que ha sido relacionada a la duración y estabilidad de la pareja (Hetherington \& Kelly, 2005).

Se debe, sin embargo, diferenciar la interdependencia positiva de los miembros de la pareja de otro concepto que es la codependencia. Según Hetherington \& Kelly (2005), la diferencia radica en que las parejas interdependientes se mantienen unidas por el amor, y las codependientes, por el miedo y la neurosis; en las parejas codependientes "el marido y la mujer son incapaces de funcionar por separado, de actuar con independencia o de mantener cada uno de ellos su propia identidad" (p. 308). La codependencia, como lo expresa Noriega Gayol (2013), es

Un problema de relación dependiente de la pareja, caracterizado por frecuentes estados de insatisfacción y sufrimiento personal, donde la mujer se enfoca en atender las necesidades de su pareja y de otras personas sin tomar en cuenta las propias; se asocia con un mecanismo de negación, desarrollo incompleto de la identidad, represión emocional y orientación rescatadora hacia los demás (pág. 2).

La dependencia emocional puede ser llamada "amor obsesivo", cuando el amor se convierte en adicción y se comienza a depender de la persona que uno ama, y llega incluso a catalogarse como una enfermedad, cuya característica principal es la falta de identidad propia (Galati, 2013; Brenlla, Brizzio y Palerma, 2009, citado en Mass et al., 2011.)

Se trata entonces de una condición muy compleja con múltiples consecuencias: genera dolor en la vida del codependiente y en su familia (Noriega, 2013; May, 2000), daños físicos y emocionales, afectaciones a nivel social y laboral. Los codependientes creen que amar es poseer u ofrecerlo todo, justificando las conductas de la pareja de manera idealizada y suelen tener celos irreales ante la ansiedad de perderle (Massa, Pat, Keb, Canto, \& Chan, 2011). El mal uso del poder emocional dentro de una familia genera una dinámica insana que debilita al niño y provoca que todas las reacciones emocionales sean internalizadas. Esta dinámica genera grupos familiares disfuncionales que generan en la persona baja autoestima. A medida que las personas crecen, buscan sentirse valoradas y para lograrlo recurren a actos extremos de dedicación hacia otros, pues piensan que su vida adquirirá sentido si la dedican a cuidar del bienestar de otros (Quinnt, 2012).

Este problema tiende a prevalecer más en mujeres, por causa sobre todo de la influencia cultural de los roles de género estereotipados, que afectan significativamente la conducta y actitu- 
des al respecto (Appel, 1991, citado en Noriega 2013). Si bien la literatura no lo liga a una etapa específica del ciclo vital, se debe tomar en cuenta la posibilidad de una mayor vulnerabilidad a las relaciones de pareja codependientes en personas jóvenes. En efecto, las personas que se encuentran en la juventud recién adquirieron en el período anterior la capacidad de evaluar riesgos y tomar decisiones conscientes; los jóvenes no suelen identificar conductas de abuso psicológico; minimizan las situaciones y pasan por alto los comportamientos de control en la pareja, creando el ambiente idóneo para un patrón de relación codependiente (Gálligo Estévez, 2009).
Finalmente, autores como Llanova Uribelarrea \& Méndez Carrillo (2012), manifiestan que este tipo de trastorno puede presentar comorbilidad con cuadros de violencia de pareja, que incluye violencia física, psicológica, sexual y otras categorías como el maltrato económico o financiero; el maltrato estructural que hace referencia a las relaciones de poder, imposiciones y toma de decisiones que generan y legitiman la desigualdad; el maltrato espiritual que violenta las creencias de la víctima o le obliga a adoptar otras; y el maltrato social que implica la mutilación de las relaciones sociales de la víctima o la humillación de esta en espacios sociales.

\section{BIENESTAR PSICOLÓGICO Y CODEPENDENCIA}

De esta manera, en la presente investigación se pretendió relacionar los conceptos de bienestar mental y de codependencia, en el sentido de que las personas con buenos niveles de bienestar psicológico tendrían una menor tendencia a estar insertos en relaciones de codependencia. Puede resultar bastante obvio que las personas que están insertas en relaciones sociales positivas (de pareja u otras), van a tener niveles de bienestar psicológico superiores. Sin embargo, la relación inversa ya ha sido también demostrada en psicología social. Así como lo afirman Jalavoy, Páez y Rodríguez (2009), citando a Fredrickson (1998, 2004), entre los beneficios de la felicidad está el hecho de que esta "ensancha nuestra capacidad intelectual y potencia nuestros recursos físicos, psicológicos y sociales" (p. 285). Sentirse bien hace que las personas sean más altruistas y evalúen más positivamente las conductas de los demás, y genera consecuencias positivas a nivel físico (longevidad), intelectual (aumento de la creatividad), psicológico (mayor resiliencia) y social (mejoría de las relaciones con los demás). 
Desde esta línea podemos entonces presuponer que una persona con mejor bienestar psicológico será una persona que entablará mejores relaciones personales, incluida las de pareja.

\section{METODOLOGÍA}

\section{Participantes}

Para esta investigación se trabajó en función de una muestra $(n=549)$ compuesta por estudiantes de grado de todas las Facultades de la Pontificia Universidad Católica del Ecuador - Quito. Para la obtención de la muestra se utilizó la siguiente fórmula:

$$
n=\frac{N^{*} Z_{a}^{2} p^{*} q}{d^{2} *(N-1)+Z_{z}^{2}{ }^{*} p^{*} q}
$$

Ecuación 1: Fórmula para la obtención de la muestra

En su aplicación se estableció un nivel de confianza "Z" de 95 \% y un error permitido "d" igual al $5 \%$.

Debido a que la distribución, por volumen, en las facultades difería mucho entre ellas, se mantuvo la proporcionalidad en la muestra, dando como resultado la distribución de la Tabla 1. 
Tabla 1: Distribución de la muestra por Facultad o Escuela

\begin{tabular}{|c|r|r|}
\hline Facultad o Escuela & $\begin{array}{c}\text { Total de } \\
\text { estudiantes }\end{array}$ & Muestra \\
\hline Bioanálisis & 277 & 19 \\
\hline Gestión social & 88 & 9 \\
\hline Arquitectura & 904 & 27 \\
\hline Administración & 1358 & 82 \\
\hline Ciencias de la Educación & 412 & 21 \\
\hline Ciencias Exactas & 246 & 15 \\
\hline Filosofía y Teología & 112 & 5 \\
\hline Ciencias Humanas & 991 & 60 \\
\hline FCLL & 645 & 41 \\
\hline Economía & 473 & 30 \\
\hline Enfermería & 577 & 34 \\
\hline Ingeniería & 952 & 58 \\
\hline Jurisprudencia & 429 & 25 \\
\hline Medicina & 1098 & 66 \\
\hline Psicología & 885 & 57 \\
\hline TOTAL & $\mathbf{9 4 4 7}$ & $\mathbf{5 4 9}$ \\
\hline
\end{tabular}

Con un proceso aleatorio simple se escogió a los estudiantes de cada unidad académica de acuerdo con el cuadro anterior.

\section{Instrumentos}

El proyecto contempló la aplicación de dos instrumentos, cada uno de los cuales será descrito a continuación:

Escala de Bienestar Psicológico (EBP) (Sánchez-Cánovas, 2013): es un cuestionario de auto aplicación, que consta de cuatro subescalas: bienestar psicológico subjetivo, bienestar material, bienestar laboral y relaciones con la pareja. Las escalas pueden ser usadas por separado, lo que permite la utilización de partes del cuestionario según el objetivo de la investigación. El test posee baremos específicos para diferentes rangos de edad (entre 17-25 años, de 26-44, de 45-64 y de 65-90 años), enfatizando en el sentimiento que tiene la persona en 
la etapa vital que está atravesando. En la presente investigación, se utilizó únicamente la subescala de bienestar subjetivo, que según el autor "hace referencia a la felicidad o al bienestar, es decir, a mayor puntuación, mayor percepción subjetiva de bienestar". La subescala consta de 30 ítems redactados de manera afirmativa, a los cuales la persona contesta en una escala de tipo Likert que mide la frecuencia de la actitud o conducta: nunca, algunas veces, bastantes veces, casi siempre, siempre.

\section{Instrumento de Codependencia}

(ICOD): El instrumento de codependencia-ICOD- es un cuestionario mexicano de tamizaje, cuyo objetivo es estudiar la prevalencia de codependencia y describir los factores asociados. Está compuesto por 30 preguntas que indagan sobre percepciones y conductas de la mujer, en relación con una pareja que abusa de ella o la explota. Estos reactivos se encuentran agrupados en cuatro dimensiones conceptuales congruentes: mecanismo de negación, desarrollo incomple- to de la identidad, represión emocional y orientación rescatadora. Las respuestas van en una escala de 0 (no), hasta 3 ( mucho) y puede ser administrado en forma individual o grupal con duración de 10 minutos (Noriega Gayol, 2011, págs. 5-8).

El ICOD ha sido validado con muestras de mujeres por la prevalencia del problema. La codependencia también se manifiesta en los hombres; sin embargo, estudios previos han demostrado que factores de riesgo similares se presentan con mayor frecuencia como trastornos de la personalidad (Roeling, Koelbel y Rutgers, 1996), citado en (Noriega Gayol, 2011, pág. 5).

\section{Métodos para análisis de datos}

Los dos instrumentos descritos se aplicaron, durante los meses de mayo a octubre del año 2015, a una muestra estratificada de estudiantes de la PUCE, Quito en todas las facultades. Los estudiantes fueron escogidos al azar y sus edades fluctuaron entre 17 y 27 años, los detalles se muestran en las Tablas 2 y 3.

Tabla 2: Datos demográficos de la muestra según edad

\begin{tabular}{ccccc}
\hline & Promedio & Desviación estándar & Mínimo & Máximo \\
\hline Edad & 20,42 & 1,83 & 17 & 27 \\
\hline
\end{tabular}


Tabla 3: Datos demográficos de la muestra según género

\begin{tabular}{ccc}
\hline Género & Número & Porcentaje \\
\hline Mujeres & 334 & $60,84 \%$ \\
\hline Varones & 215 & $39,16 \%$ \\
\hline
\end{tabular}

\section{Procesamiento:}

Una vez recolectados los datos se utilizaron las herramientas disponibles en Excel con el siguiente procedimiento:

1. Se realizó un análisis descriptivo de cada variable para determinar su comportamiento.

2. Se desarrolló un análisis correlacional para determinar el tipo de relación entre las variables intervinientes: Bienestar Psicológico Subjetivo (sub-escala del EBP) y Codependencia.

Los resultados obtenidos y los análisis establecidos en los numerales anteriores, permitieron obtener algunas conclusiones sobre el comportamiento de las variables tanto de forma individual como conjunta. Esto permitió a los investigadores comparar de mejor manera los resultados del trabajo de campo, con la teoría de cada una de las variables y de los respectivos test.

\section{RESULTADOS}

El análisis descriptivo de los datos sobre bienestar psicológico subjetivo nos muestra que al comparar los resultados con los baremos del test para los rangos de edad establecidos, el promedio de esta variable se ubica en el percentil 70. Este resultado no tan satisfactorio puede deberse a que los individuos de la muestra son todos estudiantes de nivel universitario de una Universidad particular, versus aquellos con los que se establecieron los baremos del test, muestra muy variada a nivel de estudios e ingresos.

En cuanto a la variable codependencia, el instrumento empleado no tiene baremos sino que establece un "punto de corte" en una puntuación directa de 32 para discriminar casos de codependencia versus no-casos. Para analizar descriptivamente los resultados de esta variable, los investigadores decidieron establecer rangos, puesto que el test posee una escala de respuesta para 
cada ítem de la siguiente manera: No $(0$ puntos), Poco (1 punto), Regular (2 puntos) y Mucho (3 puntos). Los resultados muestran que el $40.58 \%$ no presenta codependencia, el $25.09 \%$ presenta poca codependencia, el $23.04 \%$ se encuentra en el nivel "regular" y el $11.28 \%$ está en el nivel de mucha codependencia; todo esto según los rangos propuestos. Sin embargo, si solo se toma el criterio de discriminación de Noriega Gayol (2011), que determina que los casos que pre- sentan un puntaje directo superior a 32 son considerados casos de codependencia, en el presente estudio el $45.72 \%$ de la muestra serían considerados casos de codependencia para la muestra analizada, dado a que obtuvieron un puntaje de 32 en adelante.

\section{Análisis descriptivo de las variables:}

A continuación se presenta el resumen de estadísticos en la Tabla 4:

Tabla 4: Resumen descriptivo

\begin{tabular}{|c|c|c|}
\cline { 2 - 3 } \multicolumn{1}{c|}{} & $\begin{array}{c}\text { PD Bienestar Psicológico } \\
\text { Subjetivo }\end{array}$ & PD Total ICOD \\
\hline Media & 114,40 & 31,53 \\
\hline Error típico & 0,81 & 0,68 \\
\hline Mediana & 117 & 30 \\
\hline Moda & 126 & 24 \\
\hline Desviación estándar & 18,92 & 16,04 \\
\hline Varianza de la muestra & 357,90 & 257,40 \\
\hline Curtosis & 0,61 & 0,45 \\
\hline Coeficiente de asimetría & $-0,79$ & 0,69 \\
\hline Rango & 99 & 102 \\
\hline Mínimo & 51 & 0 \\
\hline Máximo & 150 & 102 \\
\hline Suma & 62808 & 54312 \\
\hline Cuenta & 549 & \\
\hline
\end{tabular}


En la Tabla 5 se presenta el análisis de los principales estadígrafos:

\section{Tabla 5: Estadígrafos analizados}

\begin{tabular}{|c|c|c|}
\hline & PD Bienestar Psicológico Subjetivo & PD Total ICOD \\
\hline Tipo de distribución & Asimetría negativa & Asimetría positiva \\
\hline Calificación del tipo de distribución & $\begin{array}{l}\text { Este tipo de distribución se la puede cali- } \\
\text { ficar como buena dado que indica que la } \\
\text { tendencia de la muestra es hacia valores } \\
\text { altos de la variable. Esto es bueno según } \\
\text { las características esperadas en el test. }\end{array}$ & $\begin{array}{l}\text { Este tipo de distribución se la puede } \\
\text { calificar como buena dado que indica } \\
\text { que la tendencia de la muestra es hacia } \\
\text { valores bajos de la variable. Esto, según } \\
\text { lo esperado en esta prueba, es bueno } \\
\text { dado que implica menor codependencia. }\end{array}$ \\
\hline $\begin{array}{l}\text { Relación entre las medidas de tendencia } \\
\text { central }\end{array}$ & $\begin{array}{c}\text { Media < Mediana < Moda } \\
\text { Se comprueba el tipo de asimetría. }\end{array}$ & $\begin{array}{c}\text { Moda < Mediana < Media } \\
\text { Se comprueba el tipo de asimetría. }\end{array}$ \\
\hline Dispersión (desviación estándar) & 18,92 & 16,04 \\
\hline Coeficiente de variación & $16,54 \%$ & $50,88 \%$ \\
\hline Calificación de la variación & $\begin{array}{l}\text { Aunque la Desviación estándar es mayor } \\
\text { que la otra variable, el BPS está dentro } \\
\text { de valores aceptables de dispersión. }\end{array}$ & $\begin{array}{l}\text { Aunque la Desviación estándar es } \\
\text { menor que la otra variable, el ICOD tiene } \\
\text { valores muy elevados de dispersión. }\end{array}$ \\
\hline
\end{tabular}

\section{Análisis bienestar psicológico}

Para realizar un análisis específico de la variable de bienestar psicológico se establecieron tres grupos según el siguiente criterio:

Primer grupo: resultados hasta 97 puntos que son a quienes se sugeriría una intervención.

Segundo grupo: Resultados hasta
119 puntos que son personas que estarían en un nivel al que se pueden dirigir acciones preventivas.

Tercer grupo: personas con valores superiores a 119 puntos que según la teoría no presentan problemas.

El resultado en términos numéricos y su representación gráfica se muestran a continuación:

\begin{tabular}{|c|c|c|c|}
\hline Valores críticos & Frecuencia & \% acumulado & $\%$ simple \\
\hline 97 & 93 & $16,94 \%$ & $16,94 \%$ \\
\hline 119 & 216 & $56,28 \%$ & $39,34 \%$ \\
\hline y mayor... & 240 & $100,00 \%$ & $43,72 \%$ \\
\hline
\end{tabular}




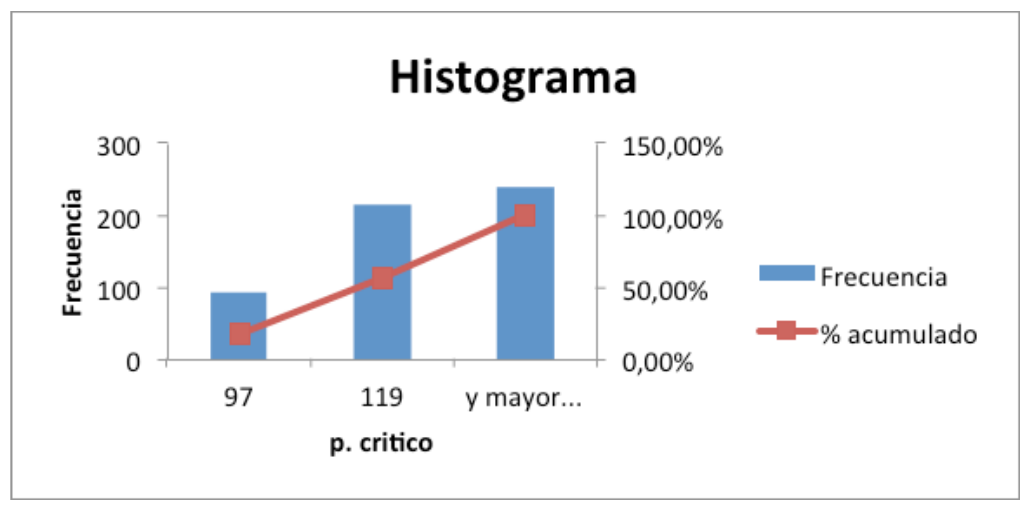

Ilustración 1: Histograma de la variable independiente.

Según estos resultados el porcentaje de estudiantes con problemas en esta variable es bastante bajo y al mismo tiempo se determina que hay un alto porcentaje de estudiantes con valores muy satisfactorios en cuanto a su nivel de bienestar psicológico subjetivo.

\section{Análisis de codependencia}

Para realizar un análisis específico de la variable de Codependencia también se establecieron tres grupos según el siguiente criterio:

Primer grupo: resultados hasta 31 puntos que según la teoría no presentan problemas.

Segundo grupo: resultados hasta 64 puntos que son personas que estarían en un nivel que requiere acciones preventivas.

Tercer grupo: personas con valores superiores a 64 puntos que son a quienes se sugeriría una intervención urgente.

El resultado en términos numéricos y su representación gráfica se muestran a continuación:

\begin{tabular}{|c|c|c|c|}
\hline Valores críticos & Frecuencia & \% acumulado & $\%$ simple \\
\hline 31 & 298 & $54,28 \%$ & $54,28 \%$ \\
\hline 64 & 232 & $96,54 \%$ & $42,26 \%$ \\
\hline y mayor... & 19 & $100,00 \%$ & $3,46 \%$ \\
\hline
\end{tabular}




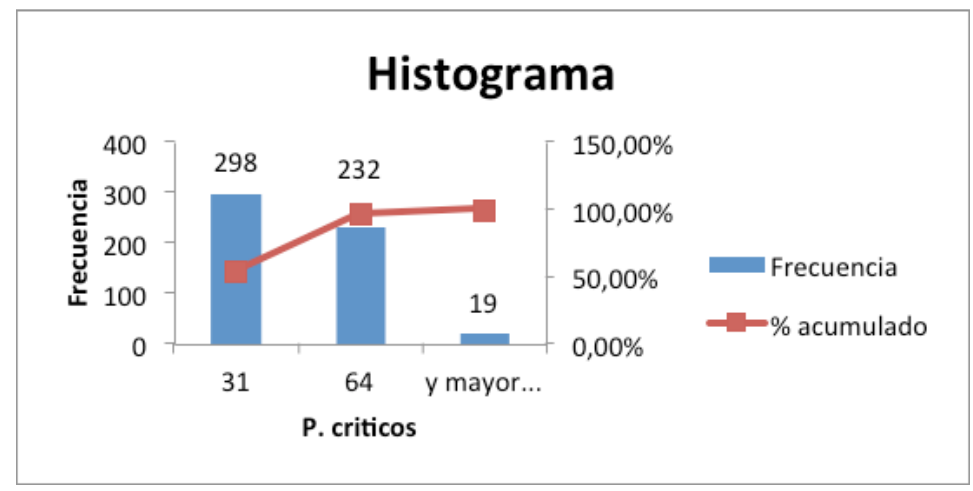

Ilustración 2: Histograma de la variable dependiente

Según estos resultados y ateniéndonos al criterio de filtro de la prueba, el porcentaje de estudiantes que no presentan relaciones con patrón de codependencia es mayoritario; lo cual es positivo para la población, pero esto no significa que el $45.72 \%$ encontrado no represente un problema en esta variable.

A pesar de que la prueba establece como punto de corte un puntaje de 32, lo cual significaría que tan solo existan dos grupos por analizar: los que cumplen esto y los que tienen valores superiores, el grupo de investigadores decidió plantear un total de cuatro grupos porque la diferencia que puede haber entre obtener 32 o 90 de hecho implica diferencias considerables en el abordaje para el trabajo con estas personas y el hacer grupos significaría tratar situaciones un poco más homogéneas en cuanto a problemática.

A continuación se presenta la propuesta antes señalada:

\begin{tabular}{|c|c|}
\hline Puntaje directo & Porcentaje por grupo \\
\hline$<32$ & $54,28 \%$ \\
\hline $32-61$ & $40,80 \%$ \\
\hline $62-91$ & $4,74 \%$ \\
\hline$>92$ & $0,18 \%$ \\
\hline
\end{tabular}




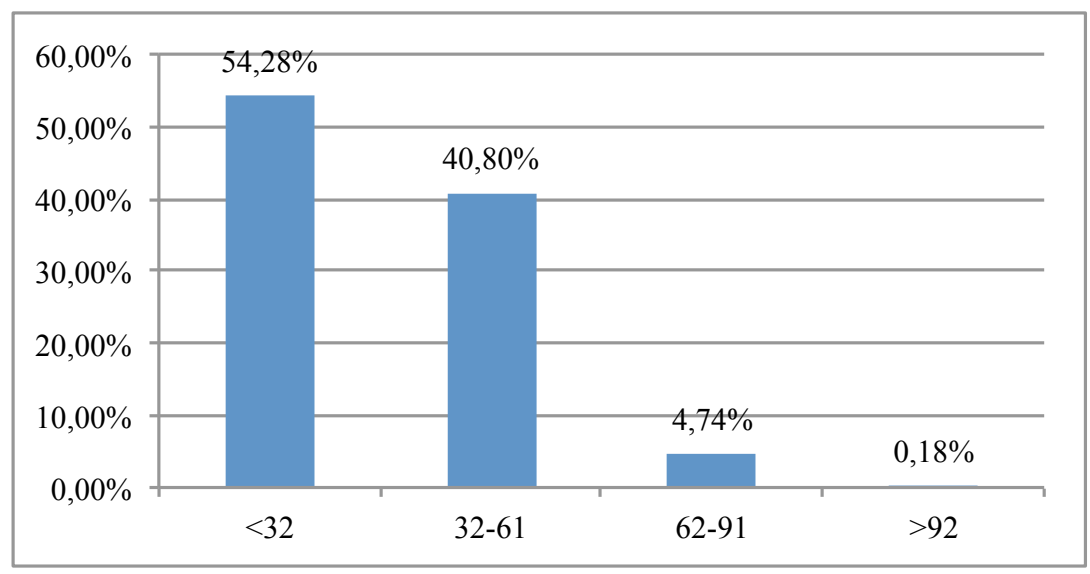

Ilustración 3: Gráfico de barras de la propuesta

Relación entre Bienestar Psicológico Subjetivo y Codependencia

Como se explicó anteriormente, el presente estudio pretendía comprobar la existencia de una relación inversa entre las variables bienestar psicológico y codependencia, en donde la primera constituye la variable independiente $(x)$ y la segunda la dependiente (y). Para el efecto, se realizó un análisis de regresión lineal simple con la función "Estimación
Lineal" de Excel cuyos resultados se presentan a continuación:

Ecuación de regresión:

$$
y=-0.367 x+73.465
$$

Error de inclinación de la recta:

$$
S_{b}=0.032
$$

Coeficiente de determinación

$$
r^{2}=0.1918(19.18 \%)
$$

\begin{tabular}{|c|c|c|}
\hline Ecuación & $-0,367001476$ & 73,46562606 \\
\hline Error de inclinación Sb & 0,032210718 & \\
\hline Coeficiente de determinación & 0,191806323 & \\
\hline
\end{tabular}

Ecuación 2: Regresión lineal. 
Nivel de confiabilidad para el análisis: $95 \%$

Hipótesis inicial Ho: no existe relación en la población; $B=0$

Hipótesis alternativa $H_{1}$ : sí existe relación en la población; $B \neq 0$

Cálculo para $B$ (población) $=b \pm Z_{t}^{*} S_{b}$

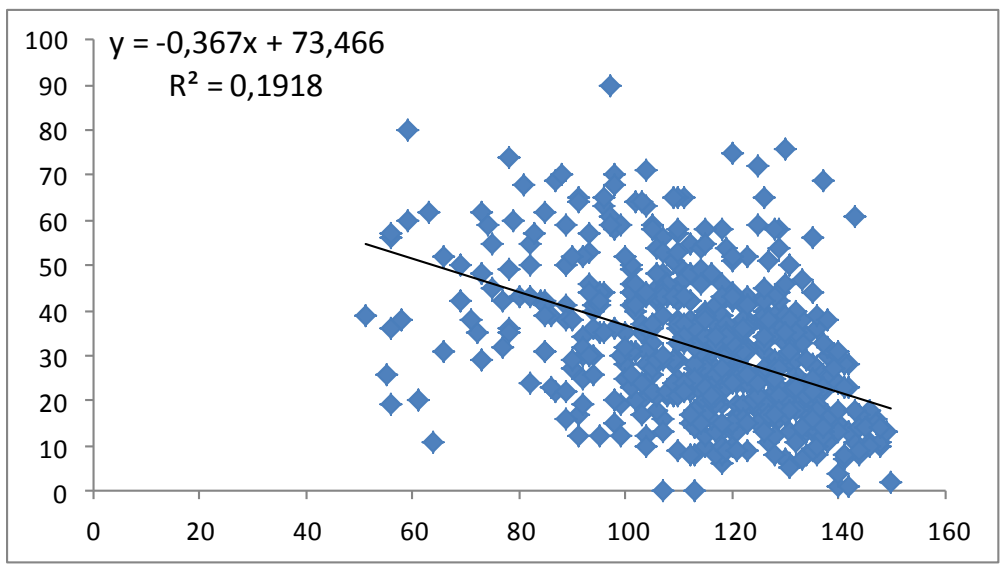

Ilustración 4: Gráfico de dispersión de las variables.

Con los resultados obtenidos se puede decir lo siguiente:

1. Se comprueba el tipo de relación esperada; es decir, el valor del coeficiente de " $x$ " en la ecuación - 0.367 indica una relación inversa entre las variables.

2. Dado que el error de inclinación es muy bajo y mucho menor que el valor del coeficiente de " $x$ ", se puede afirmar que la ecuación es consistente

3. Sin embargo, el coeficiente de determinación $\left(r^{2}=0.1918\right)$ no estable- ce una relación muy fuerte entre las variables. Esto es normal que ocurra dado que no existe la relación causa-efecto entre variables especialmente de comportamiento humano ya que cualquier variable dentro de la Psicología depende de varios factores y por tanto no es unicausal.

Para comprobar la hipótesis se encontraron los siguientes resultados:

$Z_{t}=1.96$, valor al $95 \%$ de confiabilidad

$Z_{c}=11.39$ 
Dado que el valor de $Z_{c}$ es mayor al valor de $Z_{t}$ la hipótesis inicial se rechaza; es decir, sí existe relación entre las variables; esto se comprueba también con los valores del intervalo del coeficiente de "x"en la población según lo siguiente:

$\bar{B}^{-}=-0.367-1.96 * 0.032$

$\mathrm{B}^{+}=-0.367-1.96 * 0.032$

$B^{-}=-0.4301$

$B^{+}=-0.3038$
Por lo tanto, con una confiabilidad del $95 \%$ se puede decir que el valor del coeficiente de B en la ecuación de la población ( $Y=B x+A$ ) está entre [ -0.4301; - 0.3038]; esto comprueba también que en la población se mantiene el tipo de relación inversa esperado y por tanto las conclusiones y recomendaciones que se establezcan en la muestra, pueden ser aplicadas a la población.

\section{DISCUSIÓN}

Los resultados de la investigación permiten comprobar la existencia de una relación inversa entre las variables bienestar psicológico y codependencia. Esto señala que en los sujetos de la muestra, adultos jóvenes de la PUCE, Q, aquellos que poseen un mejor bienestar psicológico subjetivo (felicidad, según Sánchez-Cánovas, 2013-) también son los que están insertos en relaciones más sanas. Inversamente, las personas con menores índices de bienestar son también aquellas que tienen más predisposición de presentar patrones de codependencia en sus relaciones de pareja; es decir, relaciones en las cuales hay insatisfacción, sufrimiento, y en las cuales los individuos se enfocan en atender las necesidades de su pareja por encima de las propias. Esto es congruente con las teorías sobre el bienestar psicológico que manifiestan que las personas felices establecen patrones relacionales positivos y enriquecedores para el individuo (Jalavoy, Páez, \& Rodríguez, 2009).

Por otra parte, también se puede observar cómo el bienestar psicológico se relaciona con las dimensiones que conforman la codependencia; pues, según lo que manifiesta Noriega Gayol (2011), los Mecanismos de negación se refieren a un mecanismo de defensa que implementa la persona codependiente para evitar experimentar una realidad difícil de enfrentar. Se manifiestan "con autoengaños y justificaciones" (p. 2), a la vez que la persona evita estar en contacto con otras personas que le podrían confrontar con la realidad; así mismo, no se establecen límites con la pareja. Ade- 
más, se encuentra íntimamente ligado a otra de las dimensiones de la codependencia que es la orientación rescatadora, debido a la cual la persona aparenta estar bien aunque se sienta mal y entre otras expresiones de esta dimensión, muestra una excesiva preocupación por los demás, evitando entrar en conflicto con ella misma (Noriega Gayol, 2011). Esto podría explicarse debido a las características de la muestra con la que se trabajó, pues las relaciones de pareja en la juventud, de acuerdo a los parámetros culturales y sociales, no suponen infelicidad o peor aún un estado de perturbación, desde este estándar es comprensible que las personas involucradas en una relación codependiente recurran a autoengaños y justificaciones que minimicen lo negativo de su realidad. Esto también podría tener su explicación en otra de las dimensiones de la codependencia que es la Represión emocional, debido a lo cual la persona involucrada en una relación de pareja con patrones de codependencia no expresa libremente sus emociones por temor a perder la aceptación de otros, especialmente de su pareja, y esta es la razón por la cual procura ser comprensiva, complacer a quienes se encuentran a su alrededor con el fin de evitar conflictos o situaciones desagradables que escapen de su control, no expresa libremente sus emociones, sentimientos o pensamien- tos por temor a perder la aceptación de los demás (Noriega Gayol, 2011). Dentro de los mismos cánones mencionados, la persona no debe tolerar malos tratos de su pareja, y sin embargo lo hace; además, evita contacto con otras personas que puedan confrontarla con su realidad, evidencia confusión y desorientación ante la situación caótica que vive y sobre todo pretende formar una imagen sana de su pareja ante los demás (Noriega Gayol, 2011).

Otra dimensión de la codependencia es el Desarrollo incompleto de la identidad, definido por Noriega Gayol (2011) como un "desarrollo infantil interrumpido por situaciones disfuncionales" (p. 2) que generará en la persona una tendencia a establecer una fuerte simbiosis con su pareja, resultándole difícil definir límites con personas abusivas y tomar decisiones "porque internamente se siente incompleta" (p. 3). Es interesante ver la concordancia de esto con lo postulado sobre la Identidad y las relaciones de pareja. Erikson (1971) enfatizó que la consecución de un grado de intimidad con otra persona demanda primero que la construcción de la identidad se haya afianzado. Parece que existe en los jóvenes de nuestra muestra un grupo importante en el cual la fusión con el otro hace que la persona se "olvide de ella misma" en la relación, descalificando sus valores y convicciones por aceptar los de su pa- 
reja, atendiendo sus necesidades en detrimento de las propias y justificando sus conductas. Muchos jóvenes se encuentran aún entonces en el tipo de relación adolescente en la cual su yo aún no está del todo definido y esto les impide lograr una verdadera intimidad. Pero si bien esto es concordante con lo teorizado por Erikson (1971), este autor sin embargo propuso que el insuficiente desarrollo de la identidad llevaría al individuo a aislarse y a no comprometerse en relaciones de pareja por el miedo a perder su identidad; sin embargo, lo que se puede observar en este estudio es que las personas con insuficiente desarrollo de la identidad no se aíslan ni entran en relaciones promiscuas, sino que entablan relaciones de pareja en donde fusionan su yo con el de su pareja, dejando que se establezcan patrones relacionales codependientes. En un análisis que este autor hace sobre "el sexo femenino y el espacio interior", postula que para los hombres la resolución de las crisis de adolescencia y juventud es muy claro (primero identidad, luego intimidad), pero en las mujeres el proceso es más complejo; por su naturaleza propia, la mujer pone su identidad a un lado cuando se prepara a definirse a través del hombre que es su pareja: "algo en la identidad de la mujer joven debe mantenerse abierto a las peculiaridades del hombre con quien se va a unir y de los hijos que va a criar" (Erikson, 1971, p.230). Este tipo de conceptualización debería ser explorada a profundidad en futuras investigaciones con muestras esencialmente femeninas, para poder sostener que la conducta codependiente es "normal" en las mujeres, puesto que la muestra del presente estudio es mixta. En todo caso, los resultados encontrados apuntan a corroborar por lo menos la deficiencia de un desarrollo de la identidad detrás de la conducta codependiente.

Una inquietud que surge frente a esto es la incógnita de si estas personas que "se pierden" en el otro, lograrán algún día completar su desarrollo de la identidad; y en ese caso, qué pasaría con la pareja, puesto que la nueva persona buscaría otro tipo de encuentro con su pareja.

En el análisis de cada una de las variables involucradas en la presente investigación, no se ha encontrado explícitamente un factor referente o asociado al tema espiritual; sin embargo, la autora May (2000) menciona que la codependencia, en última instancia, aún puede implicar la alienación de Dios, asunto que no fue evidenciado en esta investigación.

Estas preguntas pueden ser también exploradas en futuras investigaciones sobre las relaciones de pareja. 


\section{BIBLIOGRAFÎA}

Baron, R., \& Byrne, D. (2005). Psicología Social (Décima ed.). (J. L. Posadas, Ed.) Madrid, España: PEARSON EDUCACIÓN, S.A.

Bowlby, J. (1969). Attachment and loss (Vol 1: Attachment). New York: Basic Books.

Bowlby, J. (1973). Attachment and loss (Vol 2: Separation, anxiety and Anger). New York: Basic Books.

Casullo, M. M. (2002). Evaluación del bienestar psicológico. En M. E. Brenlla, Evaluación del bienestar psicológico en Iberoamérica. Buenos Aires: Paidós.

Ciccheti, D., Toth, S., \& Lynch, M. (1995). Bolwlby's dream comes full circle: The application of attachment theory to risk and psycopathology. Advances in clinical child psychology, 17, 1-31.

Craig, G. (2009). Desarrollo Psicológico, México: Pearson Educación.

Erikson, E. (1971). Identidad, juventu y crisis. Buenos Aires: Paidós.

Erikson, E. (1980). Identidad, juventud y crisis. España:Taurus ediciones.

Fernández-Abascal, E. (2009). Emociones positivas, psicología positiva y bienestar. En E. Fernández-Abascal, Emociones positivas (págs. 2761). Madrid: Ediciones Pirámide.

Fernández-Molina, M. (2015). Bienestar
Psicológico Infantil. Madrid: Ediciones Pirámide.

Galati, L. (Junio de 2013). Dependencia emocional: una aproximación al tema y su abordaje jalvoymayal tema y su abordaje desde la perspectiva cognitivo-conductual. Barcelona, España.

Gálligo Estévez, F. (2009). SOS...Mi chico me pega pero yo le quiero. Cómo ayudar a una chica joven que sufre malos tratos en su pareja. Madrid, España: Pirámide.

Hetherington, E., \& Kelly, J. (2005). En lo bueno y en lo malo: la experiencia del divorcio. Barcelona: Paidós.

Instituto Nacional de Estadísticas y Censos. (2011). Instituto Nacional de Estadísticas y Censos. Recuperado el 16 de 10 de 2014, de http:// www.inec.gob.ec/sitio_violencia/ presentacion.pdf

Jalavoy, F., Páez, D., \& Rodríguez, Á. (2009). Felicidad y relaciones interpersonales. En E. Fernández-Abascal, Emociones Positivas (págs. 277305). Madrid: Ediciones Pirámide.

Massa, J., Pat, Y., Keb, R., Canto, M., \& Chan, N. (Septiembre de 2011). La definición de amor y dependencia emocional en adolescentes de Mérida, Yucatán. Revista Electrónica de Psicología Iztacala, XIV(3), 
176-193.

May, D. (2000). Codependencia. La dependencia controladora. La dependencia sumisa. (F. Campillo Ruiz, Trad.) Bilbao, España: DESCLÉE DE BROUWER, S.A.

Noriega Gayol, G. (2011). Instrumento de Codependencia. Instrumento de Codependencia, 5-14. (P. Corona Duarte, Ed.) México, México: Manual Moderno.

Noriega, G. (2013). El guión de la codependencia en relaciones de pareja. Diagnóstico y tratamiento. México, México: El Manual Moderno.

Pérez Padilla, M.L., Ponce Rojo, A., Hernández Contreras, J. y Márquez Muñoz, B.A. (2010). Salud mental y bienestar psicológico en los estudiantes universitarios de primer ingreso de la Región Altos Norte de Jalisco. Revista de Educación y Desarrollo, 14, 31-37.
Quinnt, R. (13 de Marzo de 2012). Slideshare. Recuperado el 6 de Mayo de 2014, de http://www.slideshare. net/RachelQuinnt?utm_campaign=profiletracking\&utm_medium=sssite\&utm_source $=$ ssslideview

Ryff, C., \& Singer, B. (1998). The contours of positive human health. Psychological Inquiry, 9(1), 1-28.

Ryff, C., \& Singer, B. (1998b). The role of purpose in life and personal growth in positive human health. En P. Wong, \& P. Fry, The human quest for meaning: A Handbook of psychological research and clinical apllications (págs. 213-235). Mahwah: Lawrence Erlbaum Asoociates.

Sánchez-Cánovas, J. (2013). Escala de Bienestar Psicológico EBP (Tercera ed.). Madrid: TEA Ediciones. 